Proceedings of the International Symposium on Physics of Materials (ISPMA 14), September 10-15, 2017, Prague

\title{
High Resolution 3D Phase Field Simulations of Single Crystal and Polycrystalline Solidification
}

\author{
P. Strachota* And A. Wodecki \\ Department of Mathematics, Faculty of Nuclear Sciences and Physical Engineering, \\ Czech Technical University in Prague, Trojanova 13, 12000 Praha 2, Czech Republic
}

\begin{abstract}
We deal with numerical solution of a three-dimensional phase field model of solidification in single component anisotropic materials. In this contribution, we extend the model by crystal orientation transformation. A robust algorithm is then developed to simulate the growth of multiple grains with an arbitrary number of random crystallographic orientations and a fully resolved 3D dendritic geometry. In the first part, the model and the parallel implementation of the algorithms are explained. The second part is devoted to demonstrating the effect of meshrelated numerical anisotropy and the simulations of complex polycrystalline solidification on very fine meshes.
\end{abstract}

DOI: 10.12693/APhysPolA.134.653

PACS/topics: 81.10.Aj, 61.50.Ah, 81.30.Fb

\section{Introduction}

Phase field models are common in simulations of phase transition phenomena. The model in question [1] describes single component melt solidification with anisotropic surface tension [2, 3]. We elaborate on our existing numerical algorithm [4] and its parallel implementation [5] in order to simulate the growth of multiple crystals with random orientations. Unlike the works $[6,7]$ based on the multi-phase-field method, our approach involves a single phase field together with orientation information handled separately. While a similar work [8] uses incremental propagation of the crystal orientation as the crystal grows, our algorithm always partitions the whole computational domain according to the governing orientations and this partitioning changes dynamically during the evolution of the crystals. Crystals can develop a full dendritic structure until they meet with their neighbors to form grains. Crystal envelopes such as in $[9,10]$ are not used.

\section{Problem formulation}

The problem in the dimensionless form is posed in a domain $\Omega \subset \mathbb{R}^{3}$ and time interval $\mathcal{J}=(0, T)$ as

$$
\begin{aligned}
& \frac{\partial u}{\partial t}=\Delta u+L \frac{\partial p}{\partial t} \quad \text { in } \quad \mathcal{J} \times \Omega, \\
& \alpha \xi^{2} \frac{\partial p}{\partial t}=\xi^{2} \nabla \cdot T^{0}(\nabla p)+f(u, p, \nabla p ; \xi) \quad \text { in } \mathcal{J} \times \Omega, \\
& b_{c}(u)=0 \quad \text { on } \quad \mathcal{J} \times \partial \Omega, \\
& T^{0}(\nabla p) \cdot \boldsymbol{n}=0 \quad \text { on } \quad \mathcal{J} \times \partial \Omega, \\
& \left.u\right|_{t=0}=u_{\text {ini }},\left.\quad p\right|_{t=0}=p_{\text {ini }} \quad \text { in } \quad \Omega,
\end{aligned}
$$

\footnotetext{
* corresponding author; e-mail: pavel.strachota@fjfi.cvut.cz
}

with the boundary condition operator $b_{c}$ in Eq. (3) given by either $b_{c}(u)=u-u_{\partial \Omega}$ or $b_{c}(u)=\nabla u \cdot \boldsymbol{n}$. $u$ represents the temperature field and $p$ is the phase field. The value $p=0$ corresponds to the liquid phase and $p=1$ to the solid phase, with a smooth transition in between. The phase interface can therefore be defined by the relation $\Gamma(t)=\left\{\boldsymbol{x} \in \Omega \mid p(t, \boldsymbol{x})=\frac{1}{2}\right\}$. The model parameters involve the melting point of the material $u^{*}$, the latent heat $L$, the attachment kinetics coefficient $\alpha$, positive constants $a, b, \beta[1]$ and the parameter $\xi$ controlling the recovery of the sharp interface model [11]. The anisotropic operator $T^{0}$ (see $[12,2,13]$ ) is derived from the dual Finsler metric $\phi^{0}\left(\boldsymbol{\eta}^{*}\right), \boldsymbol{\eta}^{*} \in \mathbb{R}^{3}$ as

$$
T^{0}\left(\boldsymbol{\eta}^{*}\right)=\phi^{0}\left(\boldsymbol{\eta}^{*}\right) \phi_{\eta}^{0}\left(\boldsymbol{\eta}^{*}\right)
$$

where $\phi_{\eta}^{0}=\left(\partial_{\eta_{1}^{*}} \phi^{0}, \partial_{\eta_{2}^{*}} \phi^{0}, \partial_{\eta_{3}^{*}} \phi^{0}\right)^{\mathrm{T}}$.

The problem (1)-(5) describes the evolution of a twophase system where the solid region grows from one or more initial solid nuclei under supercooling conditions.

\subsection{Anisotropic surface energy and coordinate system transformation}

If the function $\psi: \mathbb{R}^{3} \mapsto(0,+\infty)$ represents the anisotropic surface energy depending on the normal direction to the surface $\boldsymbol{n}, \phi^{0}$ assumes the form $[14,15]$

$$
\phi^{0}\left(\boldsymbol{\eta}^{*}\right)=\left|\boldsymbol{\eta}^{*}\right| \psi(\boldsymbol{n}),
$$

where

$$
\boldsymbol{n}=-\frac{\boldsymbol{\eta}^{*}}{\left|\boldsymbol{\eta}^{*}\right|}
$$

The choice $\boldsymbol{\eta}^{*}=\nabla p$ ensures that $\boldsymbol{n}$ is the outer normal to $\Gamma$.

$\psi$ introduces anisotropy depending on the values of the components of $\boldsymbol{n}=\left(n_{1}, n_{2}, n_{3}\right)^{\mathrm{T}}$, i.e. its coordinates in the standard basis. This establishes a particular crystallographic orientation bound to the specimen coordinate system. For example, the formula for 4-fold anisotropy reads [16] 


$$
\psi(\boldsymbol{n})=1+A_{1}\left[n_{1}^{4}+n_{2}^{4}+n_{3}^{4}-6\left(n_{1}^{2} n_{2}^{2}+n_{2}^{2} n_{3}^{2}+n_{3}^{2} n_{1}^{2}\right)\right],
$$

where the coefficient $A_{1}$ specifies the anisotropy strength.

In order to change the crystallographic orientation, $\psi$ should accept the coordinates of $\boldsymbol{n}$ in a general orthonormal basis $\mathcal{B}=\left(\boldsymbol{v}_{1}, \boldsymbol{v}_{2}, \boldsymbol{v}_{3}\right)$, i.e. a vector

$$
\boldsymbol{m}=\boldsymbol{B} \boldsymbol{n} \quad \text { with } \quad \boldsymbol{B}=\left(\begin{array}{c}
\boldsymbol{v}_{1}^{\mathrm{T}} \\
\boldsymbol{v}_{2}^{\mathrm{T}} \\
\boldsymbol{v}_{3}^{\mathrm{T}}
\end{array}\right)=\left(\begin{array}{lll}
v_{11} & v_{12} & v_{13} \\
v_{21} & v_{22} & v_{23} \\
v_{31} & v_{32} & v_{33}
\end{array}\right) \text {. }
$$

This yields the formula

$$
\phi^{0}\left(\boldsymbol{\eta}^{*}\right)=\left|\boldsymbol{\eta}^{*}\right| \psi(\boldsymbol{m}),
$$

where

$$
\boldsymbol{m}=-\frac{\boldsymbol{B} \boldsymbol{\eta}^{*}}{\left|\boldsymbol{\eta}^{*}\right|}
$$

To find the form of the components of $T^{0}$, we perform the derivatives of $\phi^{0}$ in (6) and plug the components of $\boldsymbol{n}$ and $\boldsymbol{m}$ satisfying (8), (10) back in. The final formula reads

$$
\begin{gathered}
T_{i}^{0}\left(\boldsymbol{\eta}^{*}\right)=\left|\boldsymbol{\eta}^{*}\right| \psi(\boldsymbol{m})\left[\left(\sum_{k=1}^{3} \frac{\partial \psi}{\partial m_{k}}(\boldsymbol{m}) \cdot n_{i} m_{k}\right.\right. \\
\left.\left.-v_{k i} \frac{\partial \psi}{\partial m_{i}}(\boldsymbol{m})\right)-n_{i} \cdot \psi(\boldsymbol{m})\right]
\end{gathered}
$$

for $i \in\{1,2,3\}$.

\section{Numerical algorithms}

The numerical scheme for solving (1)-(5) with the original setting (7) has already been described in [4]. Its efficient MPI/OpenMP hybrid parallel implementation [5] has been the basis for further modifications introduced in this paper.

The algorithm follows the method of lines. For spatial discretization, a multipoint cell-centered finite volume scheme on uniform quadrilateral meshes is used. The fourth order Runge-Kutta-Merson solver [17] with adaptive time stepping carries out the time integration of the resulting system of ordinary differential equations. The higher order of the discretizations is chosen to reduce the numerical anisotropy caused by the mesh geometry.

\subsection{Polycrystalline solidification with arbitrary random orientations}

In order to simulate the growth of multiple crystals with different orientations, the crystallization nuclei within $\Omega$ are created in the form of spherical islands of solid. A random orthogonal matrix $\boldsymbol{B}$ is calculated for each nucleus, specifying the crystallographic orientation by means of $(9),(10)$. These matrices for all nuclei are recorded in the orientation table (OT). The number of the record in OT is called the orientation index (OI), starting from 1. A special value $\mathrm{OI}=0$ is reserved for undefined orientation, which defaults to isotropic surface energy $\psi(\boldsymbol{n})=1$.

To assign OI to each nucleus and keep track of it as the crystal grows, the orientation index field (OIF) is used in addition to the temperature field $u$ and the phase field $p$. OIF consists of the integer values of OI in all cells of the finite volume mesh. By means of OIF, the domain $\Omega$ is divided into regions where crystal growth is governed by different orientations. The generation of the initial condition for $p$ and OIF is depicted in Fig. 1.

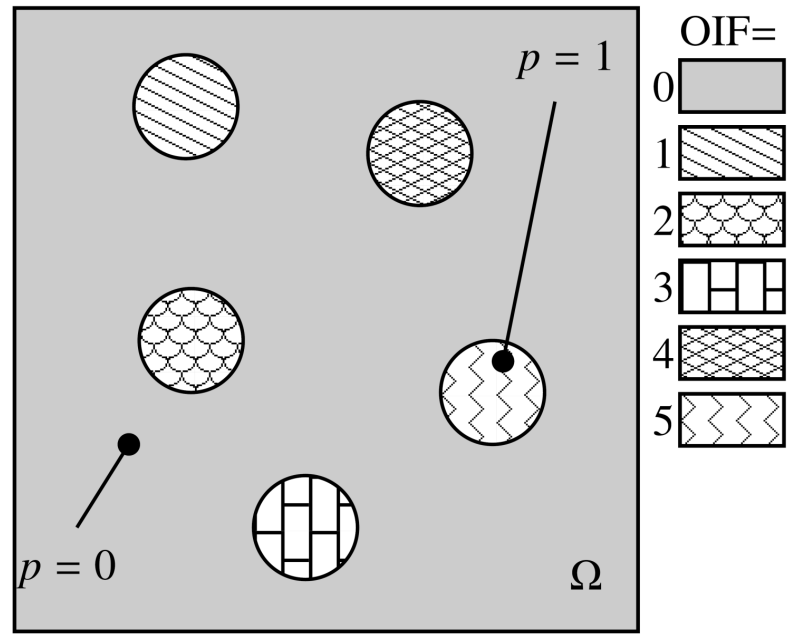

Fig. 1. The initial condition generator creates spherical solid crystallization nuclei. In the interior of each nucleus, OIF is initialized to a unique value and $p$ is set to 1 . In the rest of the domain, both $p$ and OIF are set to 0 .

The OIF is updated after each completed time step of the time integrator:

- First, OI is reset to an invalid value -1 in all cells where liquid is present (Fig. 2a).

- From each cell with an invalid OI, a path is constructed by following the gradient of $p$ (i.e. by finding the maximum of $p$ in all neighboring cells). The path ends either in a cell with a valid OI or when a local maximum of $p$ is found (Fig. $2 \mathrm{~b}$ ). The motivation is to obtain a valid OI from the nearest crystal (recall the properties of $p$ summarized in Sect. 2).

- Once the path is completed, it is filled with the found valid value of OI. In the case of the local maximum of $p, \mathrm{OI}=0$ is used instead (Fig. 2c).

As the already filled paths are never revisited, the number of steps required to fill the whole domain $\Omega$ (Fig. $2 \mathrm{~d}$ ) is proportional to the number of cells in the mesh.

\subsection{Parallel implementation of the OIF update algorithm}

The above algorithm has been implemented into the existing hybrid parallel code [5]. MPI parallelization is done by $1 \mathrm{D}$ domain decomposition into layered subdomains handled by different processes (MPI ranks). This may cause a path to span across multiple subdomains or cross a subdomain boundary more than once (Fig. 3). The solution is as follows: 

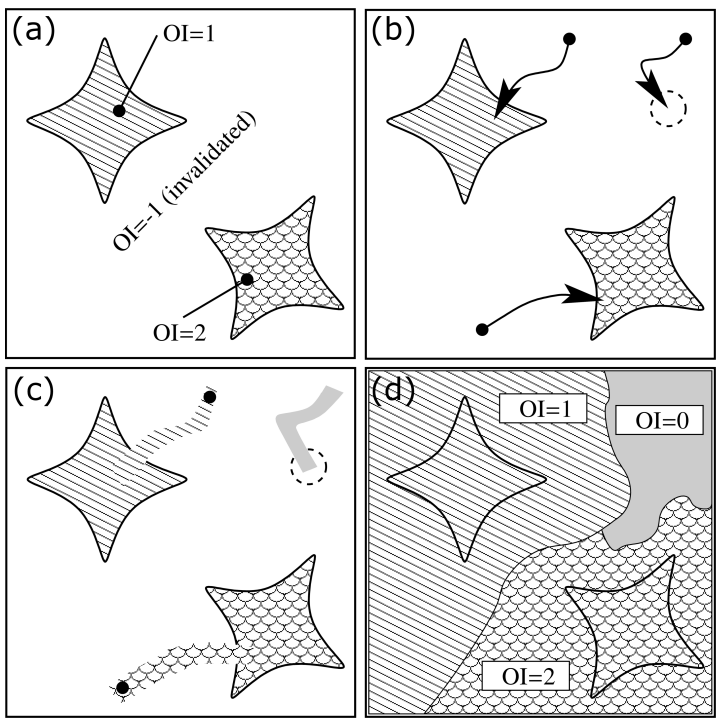

Fig. 2. Steps of the OIF update algorithm depicted in $2 \mathrm{D}$ for two crystals with different orientations. (a) Initiation phase: valid OI values are kept only in the solid, i.e. where $p>\frac{1}{2}$. (b) From each cell, a path is constructed along the gradient of $p$ until a valid OI value or a local maximum of $p$ is reached. (c) The path is filled with the found value of OI. In the case of a local maximum of $p, \mathrm{OI}=0$ (isotropic surface energy) is used. (d) After all cells are traversed, the OIF contains valid nonnegative integers again. $\Omega$ is divided into regions governed by different orientations.

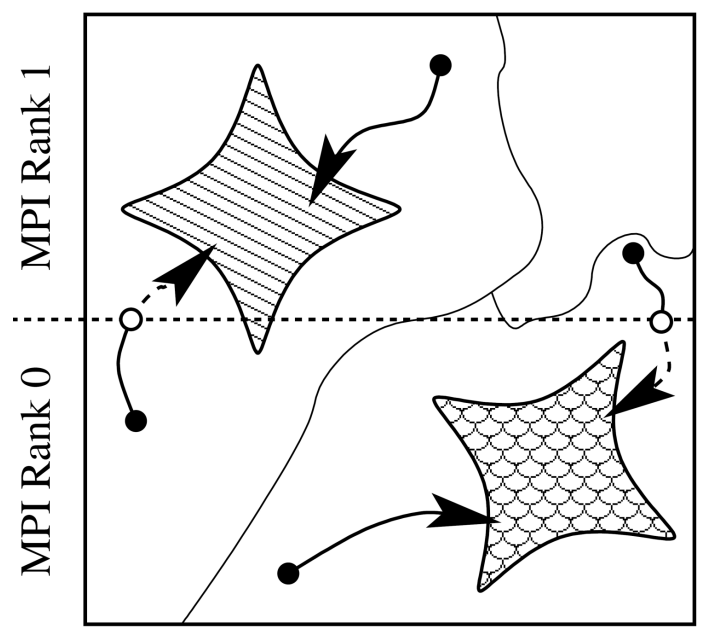

Fig. 3. Paths crossing the subdomain boundary in the case of two processes (shown in 2D).

1. First, the path finding algorithm as described above is performed. Paths leading across subdomain boundaries are temporarily filled with pointers to the cells in the neighboring boundary layers instead of OI.

2. Each process receives the OIF boundary layer from one or both of its neighbors if there is at least one pointer to a cell in that layer.

3. Pointers are replaced by the received OI values if possible.
4. Steps 2 and 3 may repeat several times for each process pair until all cells contain valid OI values.

Note that in MPI, both processes taking part in the communication must cooperate. Aside from the decision to receive, each process determines whether to send data to its neighbors based on the existence of inbound paths across the boundary and the status of OIF in its own subdomain. The two extra layers of the phase field $p$ transferred from the neighbors for the purposes of the finite volume scheme are used with advantage.

Hybrid MPI/OpenMP parallelization is achieved by simply parallelizing the for loops that traverse the computational subdomain. In contrast to the conclusion of Sect. 3.1, an additional overhead arises from the fact that overlapping paths with different starting positions may be constructed by concurrent threads.
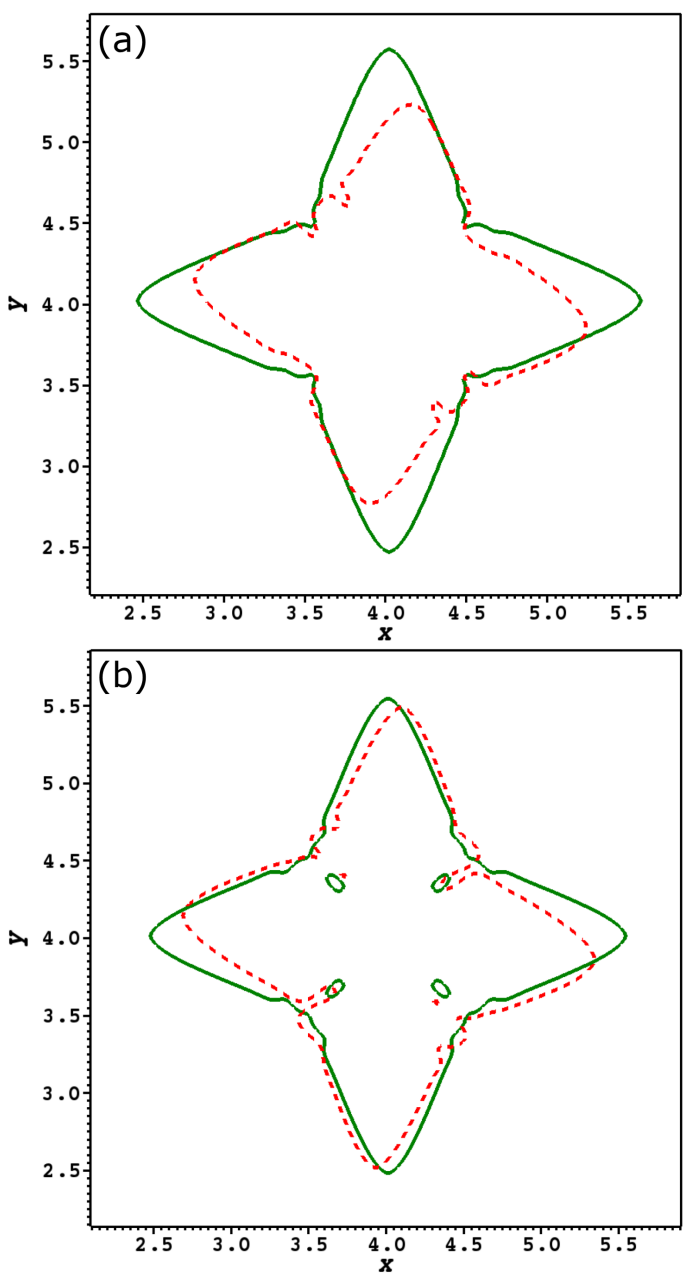

Fig. 4. Effect of the numerical mesh simulations with common parameters listed in Fig. 5 and a single crystallization seed centered at $(4,4,4)$. Slice through the crystal surface by the plane $z=4$. The solid line corresponds to the original orientation given by (7), the dashed line to the crystal rotated by $\pi / 4$ around the $z$ axis and then aligned back to the original axes after visualization. Simulations on meshes with $200^{3}$ (a) and $400^{3}$ (b) cells. 

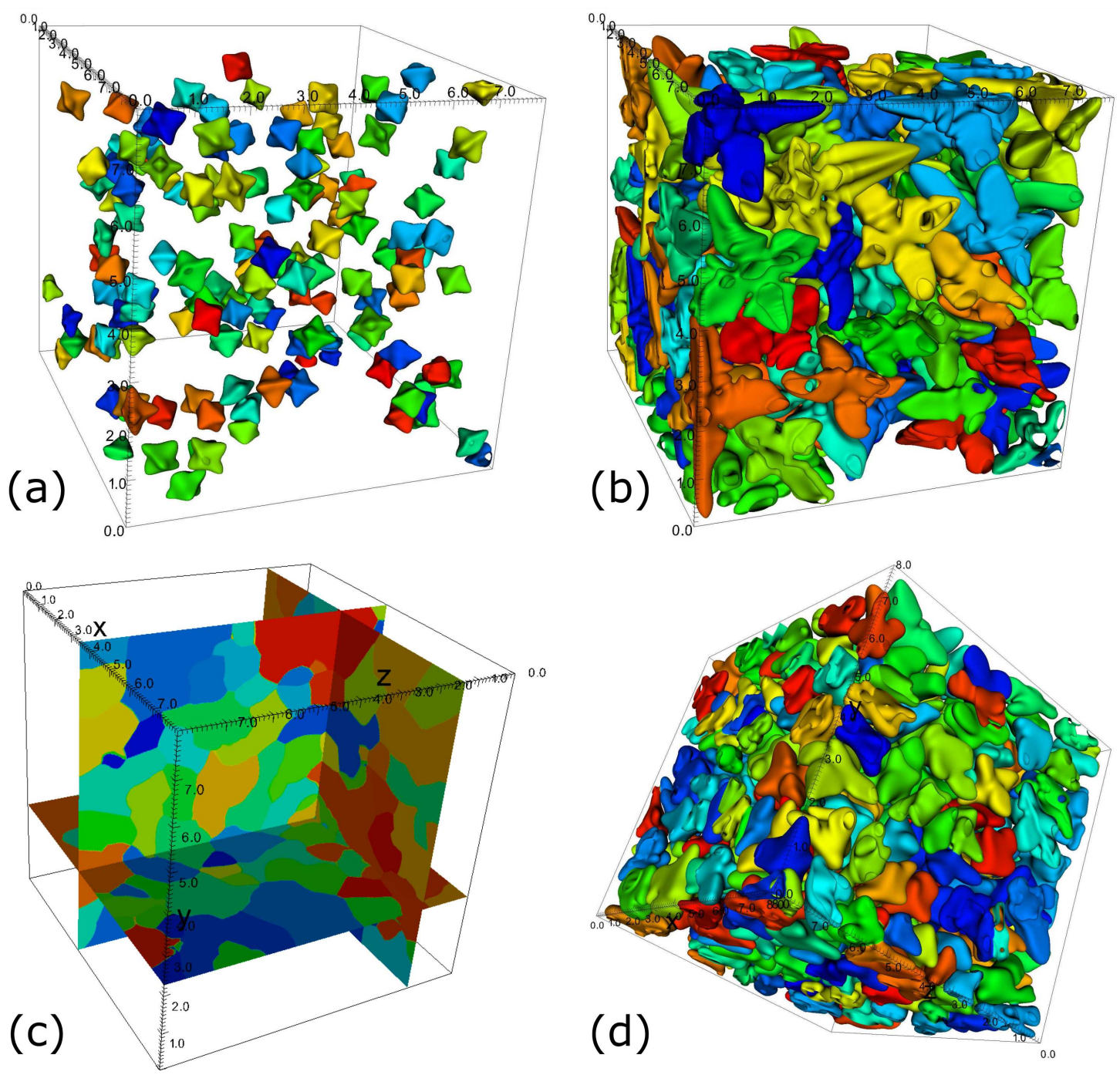

Fig. 5. Simulation results for the parameters $\Omega=(0,8)^{3}, a=2, b=1, \alpha=3, \beta=300, L=2, u^{*}=1, A_{1}=0.05$, $\xi=0.011$. $u_{i n i}=0,\left.\nabla u \cdot \boldsymbol{n}\right|_{\partial \Omega}=0$, and crystallization nucleus size 0.05 . The crystals are colored by the value of OIF. (a) Growth of 150 grains on a mesh with $500^{3}$ cells, $t=0.02$. (b) Growth of 150 grains on a mesh with $500^{3}$ cells, $t=0.12$. (c) Slices through OIF for the situation in Fig. 5b. (d) Growth of 500 grains on a mesh with $640^{3}$ cells, $t=0.13$.

\section{Results}

First, we briefly verify the orientation transformation explained in Sect. 2.1 in conjunction with the used numerical method. In Fig. 4, sample results for a crystal with and without an applied transformation are shown. The transformed crystal is drawn and the resulting shape is transformed back to the original orientation. In the ideal case, there should be no difference between the two. From the obtained results, it is obvious that the numerical scheme and the used mesh geometry causes a distortion of the crystal shape. However, this effect diminishes with increasing mesh resolution.

In Fig. 5, several results are shown to demonstrate the capabilities of polycrystalline solidification simulations. The crystal surfaces and the domain partitioning by means of OIF are presented for meshes with up to $640^{3}$ cells. To obtain the result in Fig. 5d, the MPI-parallelized simulation took $26 \mathrm{~h}$ on 4 compute nodes with 216 -core AMD Opteron 6272 CPUs (128 cores in total).

\section{Conclusion}

We present a robust and efficient parallel computational algorithm for the simulation of polycrystalline solidification based on a single phase field equation and the orientation (index) field, which is dynamically updated in the whole domain during the growth of the crystals. The regions of constant orientation always adapt to the shape of the crystals unless the crystals with different orientations merge. The algorithm is therefore suitable for the initial stage of grain boundary creation or long term simulations of dendrite coarsening provided that the supercooling is low enough. 
The challenges for further development include a realistic setting of the parameters in the dimensionless model and the choice of an appropriate mesh resolution with respect to the correct length scale. The possibility of grain consumption simulation as in $[6,7]$ and the extraction of statistical data (average grain size etc.) are also of interest.

\section{Acknowledgments}

This work has been supported by the project $M u l$ tidisciplinary research centre for advanced materials - the Center of Excellence No. 14-36566G of the Czech Science Foundation, 2014-2018, and grant No. SGS17/194/OHK4/3T/14 of the Grant Agency of the Czech Technical University in Prague.

\section{References}

[1] M. Beneš, Acta Math. Univ. Comenianae 70, 123 (2001).

[2] M. Beneš, in: Free Boundary Problems: Theory and Applications II Vol. 14, 2000, p. 18.

[3] P. Strachota, Ph.D. Thesis, Czech Technical University in Prague, 2012.

[4] P. Strachota, M. Beneš, in: Proc. ENUMATH 2011, Eds. A. Cangiani, R.L. Davidchack, E. Georgoulis, A.N. Gorban, J. Levesley, M.V. Tretyakov, Springer, Berlin 2013, p. 459.
[5] P. Strachota, M. Beneš, in: Proc. ALGORITMY 2016, 20th Conf. on Scientific Computing, Eds. A. Handlovičová, D. Ševčovič, Comenius University, Bratislava 2016, p. 23.

[6] Y. Suwa, Y. Saito, Mater. Trans. 44, 2245 (2003).

[7] Y. Suwa, Phase-Field Simulation of Grain Growth, Nippon Steel, Technical Report 102-5, Nippon Steel, 2013.

[8] Z. Guo, S.M. Xiong, IOP Conf. Ser. Mater. Sci. Eng. 84, 012067 (2015).

[9] I. Steinbach, C. Beckermann, B. Kauerauf, Q. Li, J. Guo, Acta Mater. 47, 971 (1999).

[10] M. Založnik, Y. Souhar, C. Beckermann, H. Combeau, in: Frontiers in Solidification, TMS MPMD Symposium in Honor of Michel Rappaz, Eds. W. Kurz, J. Dantzig, A. Karma, J. Hoyt, EPFL Materials Science, Lausanne 2016, p. 59.

[11] M. Beneš, Appl. Math. Czech. 48, 437 (2003).

[12] G. Bellettini, M. Paolini, Hokkaido Math. J. 25, 537 (1996).

[13] M. Beneš, Acta Math. Univ. Comenianae 76, 39 (2007).

[14] R.E. Napolitano, S. Liu, Phys. Rev. B 70, 214103 (2004).

[15] M.E. Gurtin, Thermomechanics of Evolving Phase Boundaries in the Plane, Oxford Mathematical Monographs, Oxford University Press, 1993.

[16] M. PunKay, in: NNIN REU Research Accomplishments, University of Michigan, 2005, p. 116.

[17] J.C. Butcher, Numerical Methods for Ordinary Differential Equations, 2nd ed., Wiley, Chichester 2008. 